\title{
BANDWIDTH MEASUREMENT IN WIRELESS NETWORKS
}

\author{
Andreas Johnsson, Bob Melander, and Mats Björkman \\ The Department of Computer Science and Electronics \\ Mälardalen University \\ Sweden
}

\begin{abstract}
For active, probing-based bandwidth measurements performed on top of the unifying IP layer, it may seem reasonable to expect the measurement problem in wireless networks, such as ad-hoc networks, to be no different than the one in wired networks. However, in networks with 802.11 wireless links we show that this is not the case.

Our experiments show that the measured available bandwidth is dependent on the probe packet size (contrary to what is observed in wired networks). Another equally important finding is that the measured link capacity is dependent on the probe packet size and on the cross-traffic intensity.

The study we present has been performed using a bandwidth measurement tool, DietTopp, that is based on the previously not implemented TOPP method. DietTopp measures the end-to-end available bandwidth of a network path along with the capacity of the congested link.
\end{abstract}

Keywords: Available bandwidth, link capacity, measurement, testbed, TOPP, wireless

\section{Introduction}

Wireless networks, used when connecting to the Internet or when several nodes want to communicate in an ad-hoc manner, are becoming more and more popular. Because of the increased dependence on wireless network technology, it is important to ensure that methods and tools for network performance measurement also perform well in wireless environments. In this paper, we focus on performance measurements in terms of network bandwidth, both link bandwidth and the unused portion thereof; the available bandwidth.

Measurement of network properties such as available bandwidth in for example ad-hoc networks are important for network error diagnosis and performance tuning but also as a part of the adaptive machinery of network applications such as streaming audio and video. Since the exact route between two nodes in an ad-hoc network usually is unknown and may change without no- 
tification to the application layer the end-to-end measurement of the available bandwidth should not require any infrastructure or pre-installed components at each node. To achieve that, common end-to-end bandwidth measurement methods can be applied.

State-of-the-art bandwidth measurement methods are for example Pathchirp [Ribeiro et al., 2003], Pathload [Jain and Dovrolis, 2002], Spruce [Strauss et al., 2003] and TOPP [Melander et al., 2002]. The basic principle is to inject a set of measurement packets, so called probe packets, into the network. The probe packets traverse the network path to a receiver node, which time stamps each incoming probe packets. By analyzing these time stamps estimates of the link capacity and/or the available bandwidth can be made. For many endto-end available bandwidth measurement methods no previous knowledge of the underlying network topology is needed. That is, bandwidth estimation methods are well suited for end-to-end performance measurements in ad-hoc networks. The existing methods differ in how probe packet are sent (the flight patterns) and in the estimation algorithms used (see [Prasad et al., 2003] for an overview).

In the following sections, we describe and measure bandwidth estimation characteristics when probing in 802.11 wireless networks. We show that both the measured available bandwidth and the measured link capacity are dependent on the probe packet size. Furthermore, our measurements indicate that the measured link capacity is also dependent on the cross-traffic rate.

The measurements have been performed in a testbed containing both wireless and wired hops. Our testbed topology only consist of one wireless hop, but we believe that our results illustrate the measurement problem for larger ad-hoc networks, consisting of several wireless hops, as well. To produce measurement results we have used DietTopp, a tool that measures the available bandwidth and link capacity of an end-to-end path. For comparisons and to illustrate that our observations are not tied to a certain measurement tool, we have also used the tool Pathload, that measure the available bandwidth of an end-to-end path, in our experiments.

Earlier work has touched upon the problem of active measurements of bandwidth in wireless networks. In [Johnsson et al., 2004] we discuss the main problem areas when deploying existing bandwidth measurement methods in ad-hoc networks. For example, we observed using ns-2 simulations, that the measured link capacity show dependence on the cross-traffic rate.

Measurement results presented in [Lakshminarayanan et al., 2004] indicate that the available bandwidth is dependent of the probe packet size. Our study extends that study by showing that both the available bandwidth and the measured link capacity depends on both the probe packet size and the cross-traffic rate. Further, we use a more complex measurement topology to verify their findings. 


\section{Experimental setup}

This section describes our experimental setup. That is, the measurement tool (DietTopp), our testbed and what kind of measurements we have performed and their relevance to ad-hoc networks.

\subsection{DietTopp}

DietTopp has its origins in the previously not implemented TOPP method [Melander et al., 2002]. It uses the measured dispersion of probe packet trains to calculate bandwidth estimates.

In short summary DietTopp works as follows. Starting at some offered probe rate $o_{m i n}$, DietTopp injects $m$ probe packet trains, where each train contains $k$ equally sized probe packets, into the network path. When all probe trains corresponding to a probe rate $o_{\min }$ have been transmitted, DietTopp increases the offered rate $o$ by $\Delta o$. Another set of probe packet trains are sent into the network with the new probe rate. This is repeated $i$ times until the offered probe rate reaches some specified probe rate $o_{\max }$.

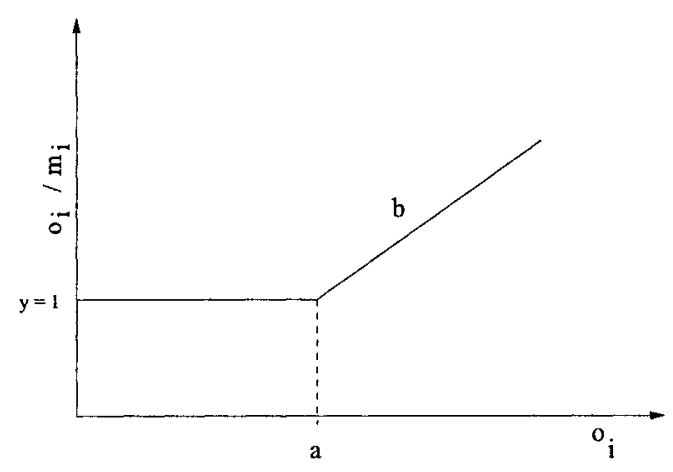

Figure l. Plot of the ratio $o_{i} / m_{i}$ as a function of $o_{i}$.

The probe packet dispersion may change as the probe packets traverse the network path between the probe sender and the probe receiver. This is due to the bottleneck spacing effect [Jacobson, 1988] and/or interactions with competing traffic.

The receiver time stamps each probe packet arrival. Hence, any change in probe packet separation can be measured. The time stamps are used to calculate the measured probe rate $m_{i}$.

When all measurements are collected, DietTopp computes the ratio $o_{i} / m_{i}$ for all $i$. If plotting the ratio $o_{i} / m_{i}$ on the y-axis and $o_{i}$ on the x-axis for all $i$, we get a plot like the theoretical one in Figure 1. If the dispersion of the probe packets would remain unchanged after traversal of the network path, 
the measured rates, $m_{i}$, on the receiver side would be the same as the offered rates $o_{i}$. Expressed differently, the ratio $o_{i} / m_{i}$ would equal 1 . The link that limits the available bandwidth of the path will eventually get congested when increasing the offered probe rate. This causes the curve to rise since the rate $m$ does not increase as much as the rate $o$. If the link capacity is $l$ and the available bandwidth is $a$ the relation between $o_{i}$ and $m_{i}$ is given by $o / m=$ $(1-a / l)+o / l$ (when one link is congested) [Melander et al., 2002].

Segment $b$ in the figure is linear and the slope corresponds to the link capacity of the congested link. The available bandwidth of the end-to-end path is defined as the intersection of $y=1$ and $b$ (i.e. $a$ in the figure) [Melander et al., 2002].

To speed up the probing phase of DietTopp we want to avoid measurements below $a$. That is, we want to ensure that $o_{\min }>a$. This is done by estimating $m_{\max }$ which is done by injecting a set of probe packets at rate $o_{\max }$ and then measure their separation at the receiver. According to [Melander et al., 2002] $m_{\max }$ is greater than the available bandwidth ( $m_{\max }$ is referred to as the asymptotic dispersion rate in [Dovrolis et al., 2001]).

Having a value of $o_{\min }>a$ the procedure described above is executed to find the link capacity and available bandwidth.

DietTopp is implemented in $\mathrm{C}++$ on Unix platforms and can be downloaded from [Johnsson, 2005].

\section{$2.2 \quad$ The testbed}

The testbed used consists of 9 computers running Linux, shown in Figure 2. The link speed for each link is shown in the figure. The links between $X w 1$, $X w 2$ and $R 1$ are $802.11 \mathrm{~b}$ wireless links (sharing the same channel) while the link between $S$ and $R 1$ either can be a $802.11 \mathrm{~b}$ wireless link or a $100 \mathrm{Mbps}$ wired link.

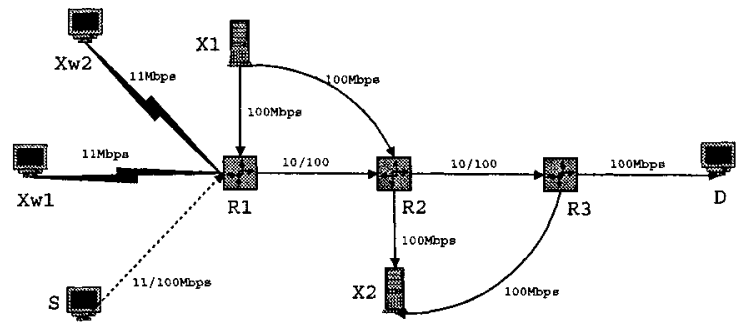

Figure 2. The testbed is constructed by one wireless link, three routers and several crosstraffic generators (on both the wireless and the wired links)

The cross traffic is generated by a modified version of $\operatorname{tg}$ [McKenney et al., 2002]. The cross traffic is either constant bit rate (CBR), exponential or pareto 
distributed (shape $=1.5)$. Further, the cross traffic consists of $60(46 \%$ of the packets), $148(11 \%), 500(11 \%)$ and $1500(32 \%)$ byte packets. This distribution of packet sizes originates from findings in [Choi et al., 2000].

\subsection{Experiments}

In this paper we want to identify possible problems associated with bandwidth measurements in wireless networks, such as ad-hoc networks.

The measurements have been performed using DietTopp. We elaborate on the impact of probe packet size, the cross-traffic distribution and on the number of cross-traffic generators in the wireless network. We compare our results to results obtained from Pathload.

This work is related to the work presented in [Lakshminarayanan et al., 2004]. We extend and complement that work in the following way: We use our newly developed tool DietTopp, that measured both the link capacity and the available bandwidth of the bottleneck link. Previous work has only focused on the available bandwidth on wireless links. Further, we use a more complex testbed scenario.

\section{Experimental results}

This section presents the results obtained from running DietTopp in different experiment scenarios. We have used Pathload [Jain and Dovrolis, 2002] to compare the obtained measurement results. In the diagrams all measurement results are shown with a $95 \%$ confidence interval.

\subsection{Measurement results in wireless networks}

This subsection presents our results from measurements using DietTopp where the bottleneck is a wireless link (the link between S and Rl in the testbed as described in subsection 2.2) which is the case in ad-hoc wireless networks. Cross traffic is present on both of the wired links R1 - R2 and R2 - R3, but the rate is limited to approximately $9 \%$ of the corresponding link capacity (100 Mbps in this case). That is, the wireless link is the link that limits both the link capacity and the available bandwidth. The cross traffic at the $100 \mathrm{Mbps}$ links between R1, R2 and R3 is pareto distributed (with respect to cross-traffic packet arrival times) and consists of 4 different packet sizes. The cross-traffic configuration on the wired links is the same for each experiment presented in this section.

The probe-packet size affects both the measured link capacity and the available bandwidth estimate when the bottleneck on an end-to-end path is a wireless link. We illustrate and describe this phenomenon in a set of diagrams below. 


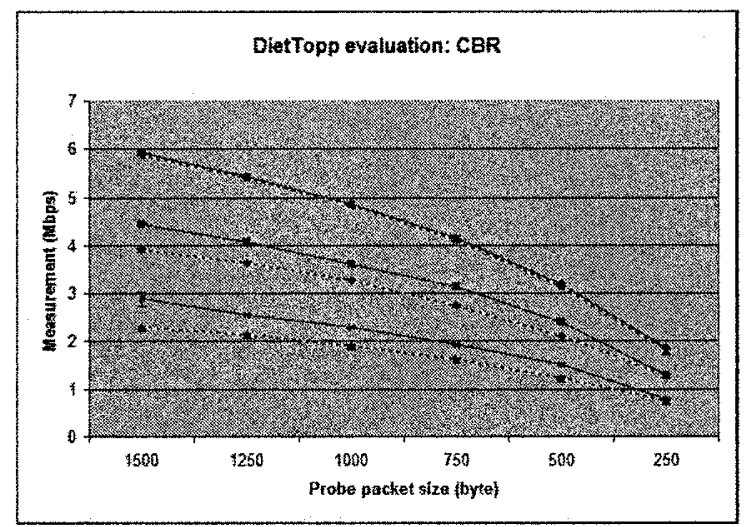

Figure 3. Available bandwidth (dashed lines) and measured link capacity (solid lines) measured under 0, $250 \mathrm{Kbps}$ and $500 \mathrm{Kbps}$ cross-traffic rates.

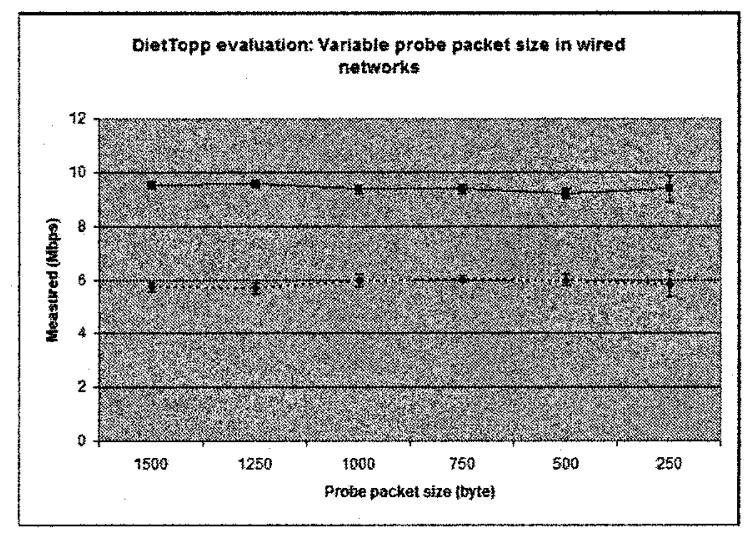

Figure 4. Available bandwidth (dashed line) and link capacity (solid line) measured by DietTopp in a wired network using different probe packet sizes. The cross traffic is a $3.26 \mathrm{Mbps}$ pareto distributed stream on a $10 \mathrm{Mbps}$ link.

The two upper curves in Figure 3 show the measured link capacity (solid line) and the measured available bandwidth (dashed line) when no cross traffic is present on the wireless link. Varying the probe packet size from 1500 bytes down to 250 bytes gives decreasing values of both the measured link capacity and the measured available bandwidth. It should be observed that the total number of bits remains constant independent of the probe packet size. The total amount of probe data sent by DietTopp in these measurements is $1.2 \mathrm{Mbit}$. Each probe train consists of 16 probe packets and we send 5 probe trains on each probe rate level. The number of probe rate levels depends on the probe 
packet size; decreasing the probe packet size increases the number of probe rate levels.

The two middle curves show measurement estimates when there is a 250 Kbps CBR cross-traffic stream on the wireless link. The two bottom curves correspond to the case when a $500 \mathrm{Kbps} \mathrm{CBR}$ stream is present. Both the measured link capacity and the measured available bandwidth decrease with decreasing probe-packet size. Another equally important behavior is that the measured link capacity decreases when increasing the cross-traffic rate. Yet another interesting phenomenon is that the difference between the measured link capacity and the measured available bandwidth tends to be smaller for small probe packet sizes. Why this is the case is a subject of further research.

For comparison we have varied the probe packet size in an all wired network. The measurement results can be seen in Figure 4. Both the measured link capacity and the available bandwidth are quite stabile, that is independent of the probe packet size.

We have also done measurements using Pathload, a tool that estimates the available bandwidth using 300 byte packets. The results obtained from using Pathload in our testbed with different cross-traffic distributions and intensities can be seen in Table 1. When comparing results obtained by Pathload (in Figure 3) to those of DietTopp we can see that Pathload reports available bandwidth measurement estimations that are in line with estimations made by DietTopp (using interpolation between packet sizes 250 and 500 bytes).

\begin{tabular}{|c|c|}
\hline Cross traffic & Measurement (Mbps) \\
\hline \hline 0 & $2.32-2.39$ \\
\hline $250 \mathrm{k}$ cbr & $1.67-1.67$ \\
\hline $250 \mathrm{k}$ exp & $1.73-1.73$ \\
\hline $250 \mathrm{k}$ par & $1.40-1.63$ \\
\hline $500 \mathrm{k}$ cbr & $0.96-0.99$ \\
\hline $500 \mathrm{k}$ exp & $0.87-0.95$ \\
\hline $500 \mathrm{k}$ par & $1.27-1.29$ \\
\hline
\end{tabular}

Table 1. Measurement results obtained from Pathload under the influence of different crosstraffic distributions.

Figures 5 and 6 report results from the same type of measurements as in Figure 3. The available bandwidth and the measured link capacity decrease with decreasing probe-packet size and increasing cross-traffic rate. However, in these two scenarios we have used more complex cross-traffic distributions. In Figure 5 we have used exponentially distributed arrival times for the crosstraffic packets while in Figure 6 we have used pareto distributed arrival times. As can be seen in both figures the confidence intervals are larger when the cross traffic is burstier. It is also obvious that the curves are less smooth compared 


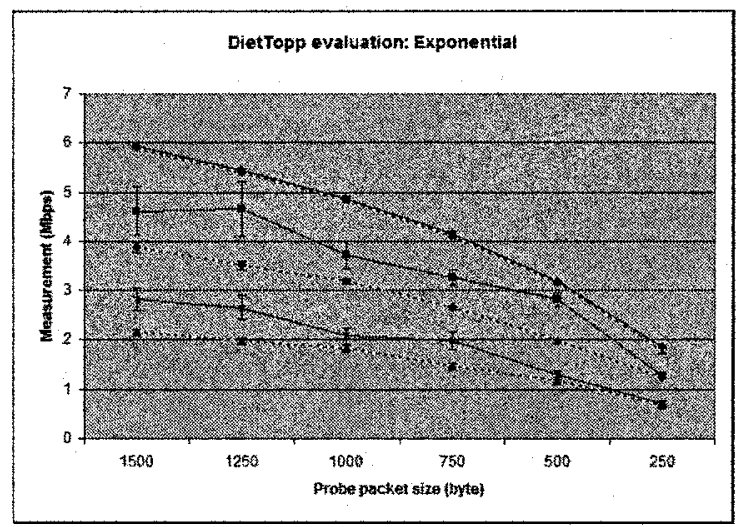

Figure 5. Available bandwidth (dashed lines) and measured link capacity (solid lines) measured under 0, $250 \mathrm{Kbps}$ and $500 \mathrm{Kbps}$ exponentially distributed cross-traffic.

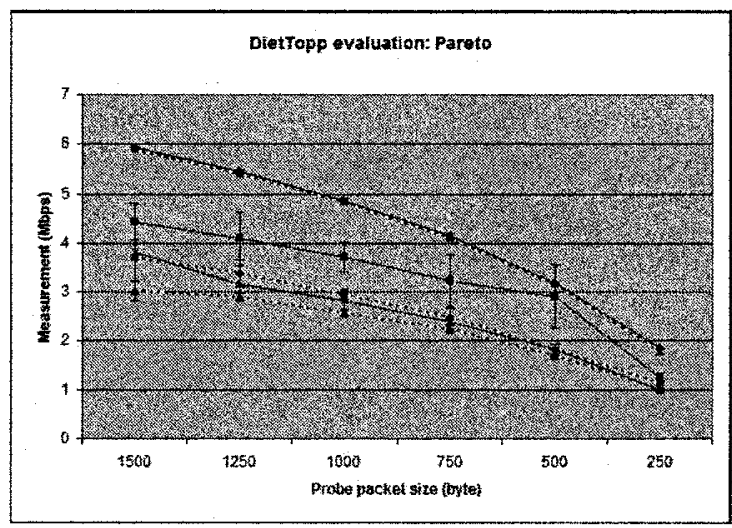

Figure 6. Available bandwidth (dashed lines) and measured link capacity (solid lines) measured under 0, $250 \mathrm{Kbps}$ and $500 \mathrm{Kbps}$ pareto distributed cross-traffic.

to the CBR case in Figure 3. In the pareto case (Figure 6) it is hard to distinguish between the $250 \mathrm{Kbps}$ and $500 \mathrm{Kbps}$ measurements of link capacity and available bandwidth. However, we can still see that the measured link capacity and available bandwidth is dependent on both the probe packet size and the cross-traffic rate. Again, comparing the measurement results (at the 300 byte probe packet size level) with results obtained by Pathload (in Table 1) we can conclude that the available bandwidth estimate characteristics are compatible.

In Figure 7 two cross-traffic generators are generating $250 \mathrm{Kbps}$ of CBR cross traffic each. Comparing Figure 7 to the measurement results in Figure 


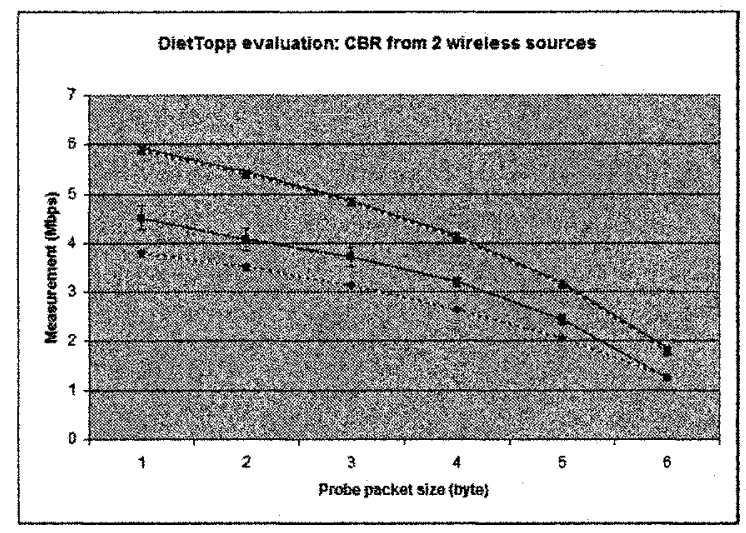

Figure 7. Available bandwidth (dashed lines) and measured link capacity (solid lines) measured under 0 and $500 \mathrm{Kbps}$ CBR cross-traffic. The cross traffic is generated by two different sources $(250 \mathrm{Kbps}$ each).

3 we see that the confidence intervals are larger when having multiple crosstraffic generators. Otherwise the curves in Figure 3 and in Figure 7 are similar.

\subsection{Wireless measurement results discussed}

The reason for the varying measurement estimates of the link capacity and the available bandwidth in our experiments (as seen in the previous section) can be derived from the link-level acknowledgments and the contention phase used in 802.11 networks. That is, if a probe packet is small, the relative overhead induced by the link-level acknowledgment and the contention phase is larger than if the probe packet were large. This will affect the probe-packet separation. In the DietTopp model the probe-packet separation is used to form estimates of both the link capacity and the available bandwidth. Hence, a smaller probe packet size will result in a lower bandwidth estimate. Due to the lack of space a more in-depth mathematical description is left out.

The results concerning the available bandwidth are in line with results discussed in [Lakshminarayanan et al., 2004]. We validate and extend the findings in [Lakshminarayanan et al., 2004] by using more complex testbed scenarios and by showing the impact of the probe-packet size and cross-traffic intensity on the measured link capacity. We also use our own tool DietTopp, that measures both the end-to-end available bandwidth and the link capacity.

\section{Conclusion}

In this paper we have shown measurements that illustrate the difference between bandwidth measurements in wired and wireless networks, such as ad- 
hoc networks. We have at a high level discussed the underlying reasons for these differences. The measurement results have been produced using our own tool, DietTopp, throughout the paper. For comparison and validity we have used Pathload. The measurements have been performed in a testbed where we have used different kinds of cross traffic, from simple CBR to bursty pareto distributed cross traffic.

Our conclusions are that measurements in wireless networks are associated with difficulties that can result in misleading bandwidth estimations. We have shown that the probe-packet size is critical to the measured link capacity and the available bandwidth. Further, we have shown that the measured link capacity on wireless links does not only depend on the probe-packet size, but also on the cross-traffic intensity.

\section{References}

Choi, Yongmin, Lee, Heung-No, and Garg, Anurag (2000). Measurement and analysis of wide area network (wan) traffic. In SCS Symposium on Performance Evaluation of Computer and Telecommunication Systems.

Dovrolis, Constantinos, Ramanathan, Parameswaran, and Moore, David (2001). What do packet dispersion techniques measure? In Proceedings of IEEE INFOCOM, pages 905-914, Anchorage, AK, USA.

Jacobson, Van (1988). Congestion avoidance and control. In Proceedings of ACM SIGCOMM, pages 314-329, Stanford, CA, USA.

Jain, Manish and Dovrolis, Constantinos (2002). End-to-end available bandwidth: Measurement methodology, dynamics, and relation with TCP throughput. In Proceedings of $A C M$ SIGCOMM, Pittsburg, PA, USA.

Johnsson, Andreas (2005). Diettopp implementation, beta version. http://www.idt.mdh.se/ãjn12/.

Johnsson, Andreas, Björkman, Mats, and Melander, Bob (2004). A study of dispersion-based measurement methods in ieee 802.11 ad-hoc networks. In Proceedings of the International Conference on Communication in Computing, Las Vegas.

Lakshminarayanan, Karthik, Padmanabhan, Venkata N., and padhye, Jitendra (2004). Bandwidth estimation in broadband access networks. In In Proceedings to the Internet Measurement Conference.

McKenney, Paul E., Lee, Dan Y., and Denny, Barbara A. (2002). Traffic generator software release notes. SRI International and USC/ISI Postel Center for Experimental Networking.

Melander, Bob, Björkman, Mats, and Gunningberg, Per (2002). Regression-based available bandwidth measurements. In Proceedings of the 2002 International Symposium on Performance Evaluation of Computer and Telecommunications Systems, San Diego, CA, USA.

Prasad, R.S., Murray, M., Dovrolis, C., and Claffy, K. (2003). Bandwidth estimation: metrics, measurement techniques, and tools. IEEE Network Magazine.

Ribeiro, Riedi, Baraniuk, Navratil, and Cottrel (2003). pathchirp: Efficient available bandwidth estimation for network paths. In Passive and Active Measurement Workshop.

Strauss, Katabi, and Kaashoek (2003). A measurement study of available bandwidth estimation tools. In ACM SIGCOMM Internet Measurement Workshop. 